\title{
Alltagsbeispiel Mittagstisch
}

\author{
Der Ausbau von Ganztagsschulen hat eine Diskussion über die Einführung \\ und Gestaltung von schulischen Mittagstischen gebracht. Sie offenbart, \\ dass bisher keine öffentliche Verantwortung für die Ernährung außer Haus \\ existiert. Fokusgruppen geben Aufschluss darüber, warum das in deutschen \\ Schulen so ist und warum ein Blick nach Schweden lehrreich sein kann.
}

I

Von Ulla Simshäuser, Gabriele Mraz und Roswitha Hofmann

m Herbst 2003 wurden vom Institut für ökologische Wirtschaftsforschung (IÖW) insgesamt sieben Fokusgruppen in der Rhein-Neckar-Region mit LehrerInnen, Eltern und SchülerInnen im Alter von 13 bis 15 Jahren zum Thema Schulverpflegung durchgeführt, vier davon in unterschiedlichen Schultypen (1). Ergänzend wurden in Einzelinterviews Rektoren und kommunale Schulträger befragt. Die beteiligten Schulen hatten bis auf die Ausnahme einer Gesamtschule bisher kaum Erfahrung mit einem schulischen Mittagstisch. Dominante Verpflegungsart waren Pausenbäcker, Supermärkte und Imbiss-Buden im Schulumfeld. Angesichts zunehmenden Nachmittagsunterrichts gab es aber in einigen Schulen erste Überlegungen und Praxisversuche. In diesem Zusammenhang interessierten wir uns für die soziale Bedeutung von Essen für die Schulakteure und für die Institution Schule.

\section{- Lebensraum Schule}

Unter den von uns befragten Schulakteuren bestand weit gehend Einigkeit darüber, dass es angesichts der zunehmenden Berufstätigkeit von Müttern, des nach wie vor geringen Engagements von Vätern und der längeren Unterrichtszeiten ein schulisches Verpflegungsangebot geben müsse. In allen Schulen waren in der Vergangenheit Projekte von engagierten LehrerInnen und Eltern durchgeführt worden. Sie führten aber bisher nicht zu einer dauerhaften Strukturbildung mit ernährungsphysiologisch sinnvollen Verpflegungsangeboten. Woran liegt es, wenn der Bedarf so evident ist?

Auf der vordergründigen Ebene fehlt die erforderliche schulische Infrastruktur, in erster Linie die Räume und das nötige Geld. Das Thema Essen in der Schule berührt darüber hinaus aber Fragen eines viel grundsätzlicheren schulischen
Selbstverständnisses, das die Gruppendiskussionen offenbarten: es geht es um Schulentwicklung und um die Frage nach der Partizipation bei der Gestaltung von schulischem Lebensraum. Die Räume fehlen nicht nur für das gemeinsame Essen, sondern auch für Bewegung und Entspannung wie etwa bewegungsfreundliche Pausenhöfe oder Gemeinschaftsräume. Von SchülerInnen und LehrerInnen wird dies aber vor allem als Zeichen der persönlichen Wertschätzung in der Institution Schule symbolisch gedeutet. Zusammen sitzen, essen und trinken, sich in zwangloser Atmosphäre austauschen und Räume selbstbestimmt nutzen und gestalten zu können, ist ein zentrales Element von Lebensqualität in der Schule, die fehlt, wie die folgende Diskussionssequenz unter Haupt- und RealschülerInnen zeigt:

„Ich finde es blöd, dass ich in der Pause im Stehen essen muss. Ich würde mich da schon lieber irgendwo hinsetzen“. ,Ja, das ist auch gemütlicher“. ,... die Lehrer in der Schule haben ihren eigenen Raum, die haben es da gemütlich in der Pause, dann gehen die mal zum Bäcker und holen sich Brötchen, die machen es sich gemütlich und wir stehen da in der Kälte. ... Ich glaube, die sehen es auch gar nicht so richtig, weil denen geht es ja gut..."

„Im Stehen“ essen zu müssen und „draußen“ zu stehen, vermittelt den SchülerInnen das Gefühl, fast schon Störfaktoren zu sein. So mutmaßten SchülerInnen in der Diskussion unter sich, dass „Chaos“ und „Dreck“ die wesentliche Gesichtspunkte seien, welche für Erwachsene gegen einen Mittagstisch sprechen. Weil SchülerInnen Dreck machen, gibt es - aus ihrer Sicht - keinen Raum für Schulverpflegung, sondern nur den Bäcker auf dem Pausenhof:

„,... deswegen haben sie ja den Schulbäcker eingestellt, dass der was bringt. Das lohnt sich irgendwie für die gar nicht, einen Computer- raum zur Verfügung zu stellen oder eine Mensa. Unsere Schule zum Beispiel würde das gar nicht machen, denn erstens würde das so dreckig sein, es würde überall Essen rumfliegen, wer soll das sauber machen? Und zweitens, im Computerraum würden dann bestimmt die Computer gleich kaputt gemacht.“

Gemeinsames Essen ist identitätsstiftend für die Schule und kann die Verbindung zwischen LehrerInnen und SchülerInnen fördern:

„Ich gehe von Tisch zu Tisch, lege mal eine Hand auf die Schulter und frage jeden, wie es schmeckt usw. Das hat an und für sich keine Bedeutung.... Aber es ist eine persönliche Ansprache, sozusagen ein kurzes ,Ich nehme Dich wahr, es ist mir nicht völlig egal, wie Du Dein Zeug da runterwürgst'“. (Lehrer an der Gesamtschule)

\section{Hidden Agenda und Demokratie}

In überraschend deutlicher Form arbeiteten die TeilnehmerInnen der Fokusgruppen diese Hidden Agenda des Themas Essen in der Schule heraus. Es geht um die Gestaltung gemeinsamen Lebens. Es geht um öffentliche Beteiligung, um Partizipation im umfassenden Sinne, um die Einschätzung der eigenen Gestaltungsmacht bei der Veränderung von Schule und Gesellschaft und es geht im erweiterten Sinne um das Selbstverständnis als künftige(r) BürgerIn. Es ist kein Zufall, dass sich gerade an einer Hauptschule dieses Bewusstsein von SchülerInnen am deutlichsten artikulierte. Ein Schüler:

„Schon der Name Hauptschule, Hauptschule ist Hauptschule, das heißt aber nicht, dass die Kinder oder die Jugendlichen....dass sie genauso ein Dreck sind, weil sie Hauptschüler sind.“ oder: ,Wenn es um Veränderungen geht, sind die Schüler ganz außen“.

Es geht darum, ob erfahren werden darf, wie Lebensfiuhrung und Umweltgestaltung in Einklang mit den körperlichen und sozialen Bedürfnissen im Alltag möglich sind, wie ein Gleichgewicht von Arbeiten und Entspannen, Sitzen und Bewegen, gutem Essen und Arbeiten im Alltag hergestellt werden kann als eine Grundlage gesundheitskompetenter Lebensführung auch im Erwachsenenalter (2).

Der Schulvergleich zeigt, dass es um einen systemischen Kontext geht, der sich in den Schulen unterschiedlich darstellt. Überwiegend wird aber Schule von den Beteiligten als veränderungsresistentes System wahrgenommen, in dem die Verantwortung für Ernährung und Gesund- 
heit von einem Akteur zum andren delegiert wird. ,Jeder sagt, es ist nicht mein Problem und schiebt es zum nächsten“, bemerkte etwa eine Lehrerin.

\section{- Zivilgesellschaftliche Verantwortung}

Kommunale Schulträger entscheiden auf Antrag und in Abstimmung mit der Schulleitung über Bau und Einrichtung von Mensen und über die Vergabe von Schulverpflegungsangeboten. Schulverpflegung ist für sie ein neues Aufgabengebiet, orientierende Qualitätsstandards für die Bewertung von Verpflegungsangeboten fehlen weitgehend. Andere kommunale Behörden wie Gesundheitsämter befassen sich jenseits der Verantwortung für die Einhaltung von Hygienerichtlinien bisher nicht mit dem Thema Schulverpflegung. Verwaltungshandeln und Ressortabgrenzung dominieren auf kommunaler Ebene. Aber auch auf Landesebene sind Kooperationen unterschiedlicher Ministerien rar. Dort wo erste Schritte unternommen werden, reagieren Kommunen zurïckhaltend, weil finanzielle Folgen bei der Festlegung von Leistungsverzeichnissen befürchtet werden.

In den Interviews mit Schulträgern war einerseits eine stark fürsorgliche Orientierung für die Schulen spürbar, angesichts leerer Gemeindekassen dominierten aber Kosten- und administrative Gesichtspunkte. Durch den Handlungsdruck hielten sich Schulträger an Beratung und Angebote von Caterern vor Ort. Marktakteure haben damit in der aktuellen Praxis die eigentliche Gestaltungsmacht über die Mittagstische. Ähnlich wie in der Schule stellt sich auch im kommunalen Bereich die Frage der Ernährungsverantwortung. Ist die Gestaltung des öffentlichen Ernährungsalltags Gegenstand schulischer, beziehungsweise kommunaler Verantwortung? Handelt es sich dabei um eine zukunftsrelevante gesellschaftliche Gestaltungsaufgabe, die von öffentlichem Interesse ist oder ist sie allein eine Frage von Verwaltungshandeln oder an Wirtschaftsakteure delegierbar?

Die Dringlichkeit dieser Fragen ist bekannt. Mit entsprechenden Appellen haben Ernährungswissenschaft und Zivilgesellschaft auf die erforderliche Qualitätssicherung in diesem Bereich aufmerksam gemacht, auf die Relevanz von Essen und Ernährung als Kulturtechnik und auf das Erfordernis von Ernährungs- und Verbraucherbildung (3). Auch die entsprechenden Qualitätskriterien sind formuliert (4). Die schlep- pende Umsetzung und die Schwierigkeiten kommunaler Praxis zeigen, dass hier ein neues Selbstverständnis von gesellschaftlicher Ernährungsverantwortung geschaffen, durch entsprechende Strukturen institutionell getragen, finanziert und öffentlich kommuniziert werden muss.

\section{- Der Blick über die Grenzen}

Beispiele aus anderen Ländern können insbesondere im Hinblick auf die Übernahme von Ernährungsverantwortung, die Finanzierung von Schulernährung und die Mitbestimmung bei der Schulverpflegung Vorbildwirkung für Deutschland haben. Zwar ist es nicht möglich, Good Practice - Beispiele aufgrund ihrer historischen und kulturellen Kontextualisierung einfach auf die Praxis anderer Länder zu übertragen, wertvolle Anregungen können sie jedoch liefern. Aufgrund seiner im internationalen Vergleich herausragenden Gleichstellungspolitik und der damit verbunden Gestaltung des Alltages von Erwachsenen und Kindern erscheint hier insbesondere Schweden interessant. Ein Blick auf die politischen und kulturellen Rahmenbedingungen zeigt, dass das Kernstïck der schwedischen Gleichstellungspolitik das Leitbild der/des durch Erwerbstätigkeit finanziell unabhängigen Schwedin/Schweden ist. Auch die Familienpolitik ist auf dieses Ziel ausgerichtet. Seit den 70erJahren werden daher berufstätige Eltern bei der Kinderbetreuung unterstïtzt. Diese Politik hat zu einem Quasi- Monopol des Staates in der Kinderbetreuung geführt. Die öffentliche Kinderbetreuung wird für Kinder im Alter von ein bis zwölf Jahren bereitgestellt, wodurch jedes Kind, unabhängig von seiner Herkunft, dasselbe Recht auf Betreuung und schulische Erziehung hat.

Das Schulgesetz in Schweden legt fest, dass jeder Schüler und jede Schülerin ein freies Essen erhalten muss. Finanziert wird das Essen aus Steuergeldern (5). Schulische Verpflegung wird bereits seit 1946 für Familien mit entsprechendem Bedarf angeboten. Zu Beginn wurde die Schulverpflegung zu 100 Prozent durch den Staat finanziert und reguliert. Im Laufe der Zeit verringerte sich der bundesstaatliche Anteil immer mehr und die Verantwortung für die Schulverpflegung verlagerte sich in Richtung Gemeinden. Seit den 70er-Jahren sind nun mehr die Gemeinden für die Bereitstellung des Schulessens verantwortlich. Zwischen den einzelnen Gemeinden existieren im Hinblick auf Qualität,
Kosten, Organisation und Zuständigkeiten Unterschiede, da die Gemeinden für diesen Zweck unterschiedlich hohe finanzielle Ressourcen bereitstellen. Im Jahr 2000 wurden durchschnittlich sechs Prozent des Gemeindebudgets für die Schulernährung verwendet.

Um Ressourcenunterschiede zwischen den Gemeinden auszugleichen, existiert in Schweden zudem ein System von Ausgleichszahlungen. So fließt etwa Geld von der reicheren Gemeinde Stockholm an ärmere Gemeinden im Norden Schwedens, was auch das Budget für Schulessen entsprechend beeinflussen kann.

Zwar ist die Bereitstellung von Schulessen wie erwähnt gesetzlich geregelt, jedoch existieren im entsprechenden Schulgesetz keine Qualitätsvorgaben. Die National Food Administration hat daher Richtlinien für die Schulernährung herausgegeben, auf die sich mittlerweile sehr viele Schulen stützen, insbesondere wenn sie diesbezügliche Leistungen zukaufen. Eine im Jahr 2001 durchgeführte Untersuchung schwedischer Gemeinden ergab, dass 46 Prozent der befragten Gemeinden politische Beschlüsse zur Verwendung von ökologisch erzeugten Lebensmitteln in ihren Großküchen getroffen haben

\section{- Der schwedische Essensbeirat}

Demokratie ist ein grundlegender Wert im schwedischen Bildungssystem. Um dem bildungspolitischen Anspruch auf Erziehung zu demokratischen Werten gerecht werden zu können, hat die schwedische Kinderbetreuung ebenso wie die Erwachsenenbildung einen dreifachen demokratischen Auftrag zu erfüllen: Den SchülerInnen sollen die fundamentalen demokratische Werte Solidarität, Gleichstellung und Chancengleichheit gelehrt werden. Die Schulen und Vorschulen selbst sollen demokratisch organisiert sein, und sie haben die Aufgabe, Mitglieder der Gesellschaft aufzuziehen, die fähig sind in einer demokratischen Gesellschaft zu leben.

Die schwedische Bildungspolitik betrachtet demnach demokratische Werte als fundamental verwoben mit organisationalen Strukturen und Ressourcen. Die Hauptverantwortung für Kinderbetreuung und Schulen liegt, wie erwähnt, bei den Gemeinden und somit sind diese verpflichtet, Ressourcen zur Verfügung zu stellen und Aktivitäten zu ermöglichen, die im Einklang mit den demokratisch grundlegenden Werten stehen. Demokratie und Gesundheitsförderung werden in Schweden ebenfalls als miteinander 
verbunden gesehen: „Learning, democracy, fundamental values and health go hand in hand" (6).

Die Relevanz für die Schulernährung ergibt sich daraus, wie zum Beispiel die Gemeinde Stockholm Mitbestimmung in der Organisation des Schulessens verankert hat. Im Rahmen der Anforderungsspezifikationen an Caterer, die das Schulessen kochen und liefern, wurde definiert, dass auf Betreiben der Firma viermal im Jahr ein so genannter Essenbeirat (Matråd) abgehalten werden muss. Dieser Essensbeirat wird besetzt aus VertreterInnen der Eltern, der SchülerInnen, der Schulangestellten, der Schuldirektion und des Caterers. Bei diesem Beirat werden eventuelle Probleme und der Bedarf an Veränderungen diskutiert. Die Firma ist verpflichtet, die auf dieser Versammlung beschlossenen Änderungen umzusetzen.

Basierend auf dem schwedischen Demokratieverständnis und der Gleichstellungspolitik haben Staat und Gemeinden Verantwortung übernommen, indem sie die Schulverpflegung finanzieren und Mitsprache ermöglichen.

\section{- Fazit}

Das Beispiel Schule zeigt: eine Ernährungswende ist voraussetzungsvoll. Sie erfordert von der Gesellschaft zunächst das Eingeständnis, dass nicht mehr nur Mütter und Familien für die Schulverpflegung verantwortlich sind und dass es ergänzend eine öffentliche Ernährungsverantwortung geben muss. In einem öffentlichen Diskurs müsste geklärt werden, wie Ernährungsverantwortung aussehen kann, welche Strukturen die Mitsprache von SchülerInnen, LehrerInnen und Eltern bei der Schulverpflegung gewährleisten und fördern.

Das Beispiel Schule zeigt auch, dass die öffentliche wie die individuelle Verantwortungsnahme für eine nachhaltige Ernährung und Gesundheit außer Haus in hohem Maße eine Demokratisierung und Humanisierung des institutionellen Alltags erfordert.

Vieles deutet darauf hin, dass Kommunen eine Schlüsselrolle bei der Veränderung nicht nur der Ernährung in Schulen haben und entsprechende Unterstützung brauchen. Eine nachhaltige Schulverpflegung kann zur Einstiegspforte für den Aufbau einer öffentlichen Ernährungsverantwortung werden. Kommunale Verantwortung für die Qualität von Schulverpflegung kann darüber hinaus zum Standortvorteil werden, sie kann Impulse geben für eine entsprechende
Entwicklung in anderen kommunalen Bereichen, wie beispielsweise einer nachhaltigen Gemeinschaftsverpflegung in Ämtern, Krankenhäusern und Pflegeeinrichtungen. Spätestens hier wird deutlich, dass die Ernährungswende in der Schule Kreise ziehen und Ernährungswenden in anderen Bereichen unterstïtzen kann.

\section{Anmerkungen}

(1) Eine ausführliche Beschreibung der Empirie findet sich ab März 2005 unter: www.ernaehrungswende.de. Vgl. auch: Holzhauer, B./Simshäuser, U.: Ernährungswende in der Schule, Herausforderungen und Chancen im Schulalltag. In: Ev. Akademie Loccum (Hg.): Der Mensch ist was er is(s)t. Tagungsbeiträge vom 16.18.4.2004, Loccum (im Erscheinen). (2) Vergleiche Ausführungen von Hayn zu Fast-Food als Ernährungsstil und Lebensphase in diesem Heft.

(3) Vergleiche die Ausführungen von Beer in diesem Heft.

(4) Stellungnahme der DGE-Arbeitskreise „Ernährung und

Schule" einzusehen unter www.dge.de/ oder Appell von Loccum einzusehen unter: www.ernaehrungswende.de

(5) Heseker et al.: Ernährung in der Ganztagsschule Teil 1, Notwendigkeit und Problematik der Ganztagsschule. DGE Arbeitskreis Ernährung und Schule. In: Ernährungsumschau, №. 3/2003, Frankfurt 2003, sowie Heseker et al.: Ernährung in der Ganztagsschule Teil 2, Institutionalisierung und Möglichkeiten von Schulverpflegung. DGE Arbeitskreis Ernährung und Schule. In: Ernährungsumschau, No. 4/2003, Frankfurt 2003.

(6) Swedish National Agency for Education (Ed.): Democracy and Fundamental Values. Stockholm 2004. Download unter: www.skolverket.se

\section{Die Autorinnen}

Dr. Ulla Simshäuser ist wissenschaftliche Mitarbeiterin in den Forschungsfeldern ökologische Produktpolitik und ökologischer Konsum des Instituts für ökologische Wirtschaftsforschung (IÖW). Gabriele Mraz ist wissenschaftliche Mitarbeiterin im Österreichischen Ökologie-Institut für angewandte Umweltforschung (ÖÖI). Roswitha Hofmann arbeitet an der Abteilung Gender and Diversity in Organizations an der Wirtschaftsuniversität Wien.

Kontakt: IÖW Heidelberg, Bergstraße 7, 69120 Heidelberg. Tel. 06221-649160,

E-Mail: Ulla.Simshaeuser@ heidelberg.ioew.de. 0̈0̈l, Seidengasse 13A1070 Wien. E-Mail: mraz@ecology.at. Uni Wien, Waldstr. 41, A-2105 Oberrohrbach.

E-Mail: Roswitha.hofmann@wu-wien.ac.at.

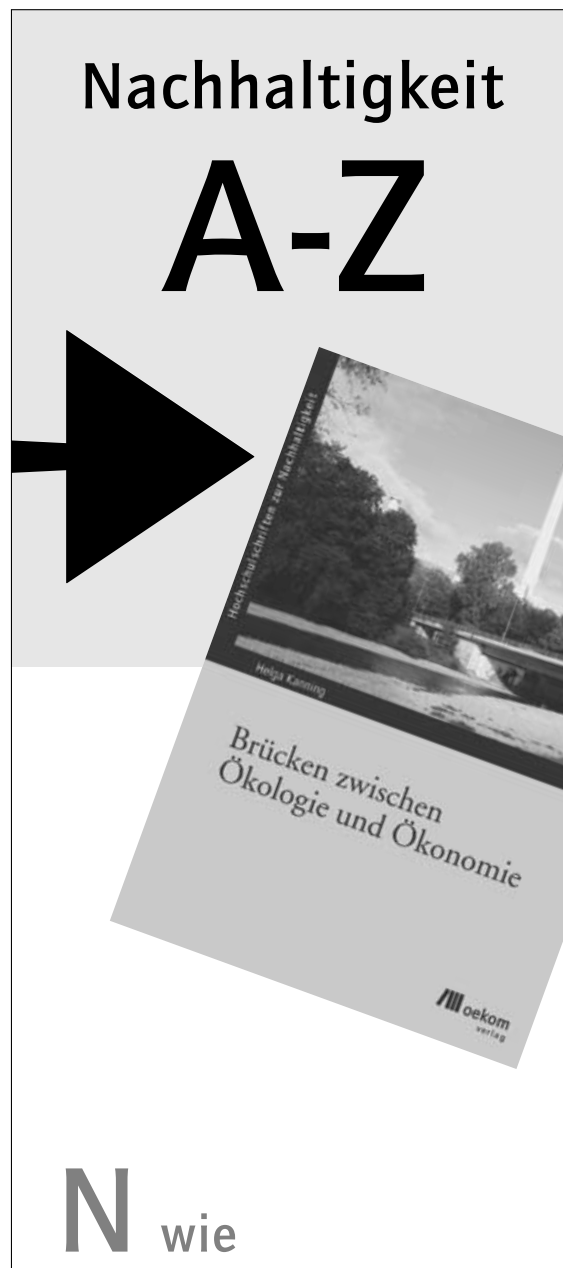

\section{nachhaltiger Gewinn}

Helga Kanning bietet neue Perspektiven für die transdisziplinäre Forschung und Entwicklung nachhaltigen Wirtschaftens. Das Buch täuscht aber nicht darüber hinweg, dass auch tiefe Gräben zwischen den Wissenschaftsdisziplinen überwunden werden müssen.

Helga Kanning

Brücken zwischen Ökologie und Ökonomie München voraussichtlich März 2005 Hochschulschriften zur Nachhaltigkeit Bd. 18 ca. 280 Seiten; $29,50 €$

ISBN 3-936581-82-7

\section{Erhältlich bei}

www.oekom.de

oekom@wmi-verlagsservice.de

Fax $++49 /(0) 8191 / 125-405$

\section{/lloekom verlag}

Die guten Seiten der Zukunft 
(c) 20I0 Authors; licensee IÖW and oekom verlag. This is an article distributed under the terms of the Creative Commons Attribution Non-Commercial No Derivates License (http://creativecommons.org/licenses/by-nc-nd/3.o/), which permits unrestricted use, distribution, and reproduction in any medium, provided the original work is properly cited. 